\title{
Boolean Logic Optimization in Majority-Inverter Graphs
}

\author{
Luca Amarú, Pierre-Emmanuel Gaillardon, Giovanni De Micheli \\ Integrated Systems Laboratory (LSI), EPFL, Switzerland.
}

\begin{abstract}
We present a Boolean logic optimization framework based on Majority-Inverter Graph (MIG). An MIG is a directed acyclic graph consisting of three-input majority nodes and regular/complemented edges. Current MIG optimization is supported by a consistent algebraic framework. However, when algebraic methods cannot improve a result quality, stronger Boolean methods are needed to attain further optimization. For this purpose, we propose MIG Boolean methods exploiting the error masking property of majority operators. Our MIG Boolean methods insert logic errors that strongly simplify an MIG while being successively masked by the voting nature of majority nodes. Thanks to the datastructure/methodology fitness, our MIG Boolean methods run in principle as fast as algebraic counterparts. Experiments show that our Boolean methodology combined with state-of-art MIG algebraic techniques enable superior optimization quality. For example, when targeting depth reduction, our MIG optimizer transforms a ripple carry adder into a carry look-ahead one. Considering the set of IWLS'05 (arithmetic intensive) benchmarks, our MIG optimizer reduces by $17.98 \%(26.69 \%)$ the logic network depth while also enhancing size and power activity metrics, with respect to $\mathrm{ABC}$ academic optimizer. Without MIG Boolean methods, i.e., using MIG algebraic optimization alone, the previous gains are halved. Employed as front-end to a delay-critical 22-nm ASIC flow (logic synthesis + physical design) our MIG optimizer reduces the average delay/area/power by $(15.07 \%, 4.93 \%, 1.93 \%)$, over 27 academic and industrial benchmarks, as compared to a leading commercial ASIC flow.

Categories and Subject Descriptors

B.6.3 [Design Aids]: Automatic Synthesis, Optimization

General Terms

Algorithms, Design, Performance, Theory.

Keywords

Majority Logic, Boolean Optimization, Logic Synthesis.
\end{abstract}

\section{INTRODUCTION}

Nowadays, EDA tools are challenged by design goals at the frontier of what is achievable in advanced technologies. In this scenario, recent logic synthesis works considered (slower) Boolean methods [1]-[5] rather than (faster) algebraic methods [6]-[9] to obtain superior circuit realizations, in terms of speed, power and area. Indeed, it is desirable to spend more time in logic synthesis computation to get a better final design. However, with traditional tools, there is a limit after which spending more effort in logic synthesis, for example running complex Boolean methods, does not improve a circuit quality or even requires too long runtime [10]. To push this limit as far as possible, innovative data structures and manipulation laws are decisive.

Majority-Inverter Graph (MIG) is a promising data structure for logic optimization and synthesis recently introduced by [11]. An MIG is a directed acyclic graph consisting of three-input majority nodes and regular/complemented edges. MIG manipulation is supported by a consistent algebraic framework. Algebraic optimization of MIGs showed strong synthesis results. However, the heuristic and local (short-sighted) nature of MIG algebraic methods [11] might preclude global (far-sighted) optimization opportunities.

In this paper, we extend the capabilities of MIG logic optimization by developing powerful Boolean methods based on majority voting. Our MIG Boolean methods enforce simplification opportunities by inserting logic errors successively masked by MIG nodes. Thanks

Permission to make digital or hard copies of all or part of this work for personal or classroom use is granted without fee provided that copies are not made or distributed for profit or commercial advantage and that copies bear this notice and the full citation on the first page. Copyrights for components of this work owned by others than ACM must be honored. Abstracting with credit is permitted. To copy otherwise, or republish, to post on servers or to redistribute to lists, requires prior specific permission and/or a fee. Request permissions from Permissions@acm.org.

DAC ' 15, June 07 - 11, 2015, San Francisco, CA, USA Copyright 2015 ACM 9781-4503-3520-1/15/06\$15.00 http://dx.doi.org/10.1145/2744769.2744806 to the data-structure/methodology fitness, our MIG Boolean methods have an efficient runtime, i.e., can handle 100k equivalent gates in less than a minute, on a standard laptop. Our Boolean methods are simple, yet powerful. Experiments combined with state-of-art MIG algebraic techniques show tremendous results. For example, when targeting depth reduction, our MIG optimizer automatically transforms a ripple carry adder into a carry look-ahead one. Considering the set of IWLS'05 (arithmetic intensive) benchmarks, our MIG optimizer reduces by $17.98 \%(26.69 \%)$ the logic network depth while also enhancing size and power activity metrics, with respect to ABC tool [13]. Without MIG Boolean methods, using MIG algebraic optimization alone, only (about) half of the aforementioned gains appeared in our experiments. Employed as front-end to a delaycritical 22-nm ASIC flow (logic synthesis + physical design) our MIG optimizer reduces the average delay/area/power by $(15.07 \%, 4.93 \%$, $1.93 \%$ ), over 27 academic and industrial benchmarks, as compared to a leading commercial ASIC flow.

The remainder of this paper is organized as follows. Section II provides a background on logic optimization and on MIGs. Section III discusses on the logic flexibility of MIGs, exploiting the intrinsic voting resilience of majority nodes. Section IV describes our Boolean optimization methodology based on MIGs. Section V shows the experimental results for our MIG Boolean optimization employed either stand-alone or as front-end to a commercial ASIC design flow. Section VI concludes the paper.

\section{BACKGROUND AND MOTIVATION}

This section gives a background on logic optimization and MIGs.

\section{A. Logic Optimization}

Logic optimization methods are usually divided into two groups: Algebraic methods, which are fast, and Boolean methods, which are slower but achieve better results [10]. Traditional algebraic methods treat a logic functions as a polynomial [6], [7]. Algebraic operations are selectively iterated over the entire logic circuits, until an improvement exists. Basic algebraic operations are extraction, decomposition, factoring, balancing and substitution [10]. Their efficient runtime is enabled by weak-division and kernel theory. Instead, Boolean methods handle the true nature of a logic function using Boolean identities as well as (global) don't cares (circuit flexibilities) to get a better solution [10], [12]. Boolean division and substitution techniques trade off runtime for better minimization quality. Most Boolean methods run on expressive data-structures, with ideally no ambiguity on the representation. Canonical logic representation forms, such as truth tables and binary decision diagrams, support efficiently Boolean methods. For example, Boolean decomposition based on binary decision diagrams can recognize re-structuring opportunities not visible by algebraic counterparts [3]-[5]. Modern optimization methodologies, and associated tools, use algebraic and Boolean methods in conjunction [9], [13], i.e., after a slow but powerful Boolean method is used fast algebraic methods are repeated until an improvement exists.

\section{B. Majority-Inverter Graph}

A Majority-Inverter Graph (MIG) is a data structure for Boolean function representation and optimization. An MIG is a logic network consisting of 3-input majority nodes and regular/complemented edges [11]. Each majority node can be reduced to a conjunction (AND) or a disjunction (OR) operator by fixing the third input to 
0 or to 1 , respectively. It follows that any AND/OR-INV graphs (AOIG) can be emulated by a structurally identical MIG. In Fig. 1, an example AOIG is depicted with its structurally, and functionally, identical MIG. However, even better MIG representations appear by

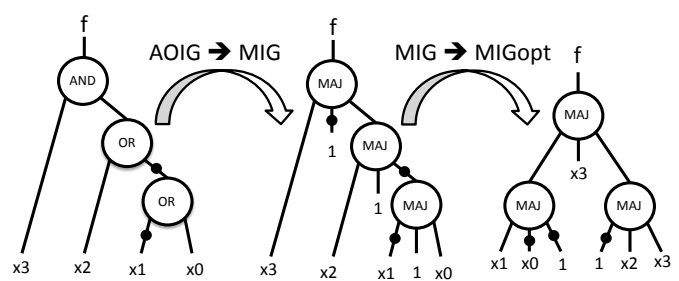

Fig. 1: Example of MIG representation.

exploiting MIG nodes functionality (majority) rather than reducing it to AND/OR. Again in Fig. 1, a more compact MIG for the same example is depicted, having one fewer level of depth and the same number of nodes. To natively optimize and reach advantageous MIGs, like the one in Fig. 1, a MIG Boolean algebra is introduced in [11] and axiomatized $(\Omega)$ by five primitive transformation rules.

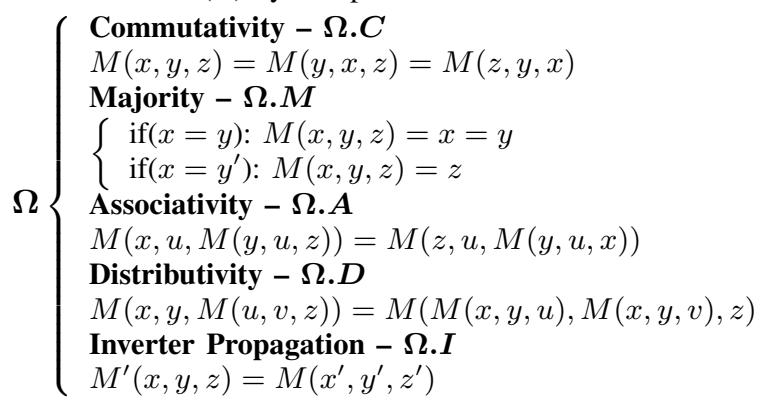

Some of these axioms are drawn from median algebra [14], [15] and others from the properties of the median operator in a distributive lattice [16]. From a theoretical perspective, it is possible to traverse the entire MIG representation space just by using a sequence of transformations drawn from $\Omega$ [11]. However, deriving such a global sequence of $\Omega$ is an intractable problem. For this reason, current MIG optimization heuristics [11] focus on local $\Omega$ transformations. We call the MIG optimization techniques in [11] algebraic, because they locally use MIG algebra transformations.

In this paper, we propose alternatives to these techniques, focusing on global properties of MIGs such as voting resilience and don't care conditions. Due to their global and general nature, we call our proposed MIG optimization methods "Boolean".

\section{HARNESSING Voting RESILIENCE IN MIG}

MIGs are hierarchical majority voting systems. One notable property of majority voting is the capability to correct various types of bit-errors. This feature is inherited by MIGs, where error masking can be exploited for optimization purposes. One way for doing so is to purposely introduce logic errors that are succesively masked by the voting resilience in MIG nodes. If such logic errors are advantageous, in terms of circuit simplifications, better MIG representations appear.

In the immediate following, we present the theoretical grounds for "safe error insertion" in MIGs, defining what type of errors, and at what overhead cost, can be introduced. Later on, we propose two intelligent procedures for "advantageous errors" insertion.

\section{A. Inserting Safe Errors in $M I G$}

Before we enter into the core theory of this work, we briefly review notations and definitions on logic errors [12], [17].

Definition The logic error between an original function $f$ and its faulty version $g$ is the Boolean difference $f \oplus g$.

In principle, a logic error can be determined for any two (potentially very different) circuits. In practical cases, a logic error is interpreted as a perturbation $A$ on an original logic circuit $f$ [12].
Notation A logic circuit $f$ affected by an error $A$ is written as $f^{A}$.

For example, considering the function $f=(a+b) \cdot c$, an error $A$ defined as "stuck variable $b$ to 0 " $(A: b=0)$ leads to $f^{A}=a c$. In general, an error flips $k$ entries in the truth table of the affected function. In the previous example, $k=1$. If $k=0$, the error is safe or permissible, as it does not change the original functionality [17].

To insert safe (permissible) errors in an MIG we consider a root node $w$ and we triplicate it. In each version of $w$ we introduce logic errors heavily simplifying the MIG. Then, we connect back the three faulty versions of $w$ to a top majority node exploiting the error masking property. Unfortunately, a majority node cannot mask all types of errors. This limits our choice of permissible errors. Orthogonal errors, defined ${ }^{1}$ hereafter, fit with our purposes. Informally, two logic errors are orthogonal if for any input pattern they cannot happen simultaneously.

Definition Two logic errors $A$ and $B$ on a logic circuit $f$ are said orthogonal if $\left(f^{A} \oplus f\right) \cdot\left(f^{B} \oplus f\right)=0$.

To give an example about orthogonal errors consider the function $f=(a+b) \cdot c$. Here, the two errors $A: a+b=1$ and $B: c=0$ are actually orthogonal. Indeed, by simple logic simplification, we get $(c \oplus f) \cdot(0 \oplus f)=\left(((a+b) c)^{\prime} c+((a+b) c) c^{\prime}\right) \cdot((a+b) c)=$ $((a+b) c)^{\prime} c \cdot((a+b) c)=0$. Instead, the errors $A: a+b=1$ and $B$ : $c=1$ are not orthogonal for $f$. Indeed, for the input pattern $(1,1,1)$ both $A$ and $B$ happen.

Now consider back a generic MIG root $w$. Say $A, B$ and $C$ three pairwise orthogonal errors on $w$. Being all pairwise orthogonal, a top majority node $M\left(w^{A}, w^{B}, w^{C}\right)$ is capable to mask $A, B$ and $C$ errors restoring the original functionality of $w$. This is formalized in the following theorem.

Theorem 3.1: Say $w$ a generic node in an MIG. Say $A, B$ and $C$ three pairwise orthogonal errors on $w$. Then the following equation holds: $w=M\left(w^{A}, w^{B}, w^{C}\right)$

Proof: We show that $w \oplus M\left(w^{A}, w^{B}, w^{C}\right)=0$. First, the $\oplus(\mathrm{XOR})$ operator propagates into the majority operator as $w \oplus$ $M\left(w^{A}, w^{B}, w^{C}\right)=M\left(w^{A} \oplus w, w^{B} \oplus w, w^{C} \oplus w\right)$. Recalling that $M(a, b, c)=a b+a c+b c$ we rewrite the previous expression as $\left(w^{A} \oplus w\right) \cdot\left(w^{B} \oplus w\right)+\left(w^{A} \oplus w\right) \cdot\left(w^{C} \oplus w\right)+\left(w^{B} \oplus w\right) \cdot\left(w^{C} \oplus w\right)$. As $A, B$ and $C$ are pairwise orthogonal, we have that each term is 0 , so $0+0+0=0$. So, $w \oplus M\left(w^{A}, w^{B}, w^{C}\right)=0$. Q.E.D.

Note that an MIG $w=M\left(w^{A}, w^{B}, w^{C}\right)$ can have up to three times the size and one more level of depth as compared to the original $w$. This means that simplifications enabled by orthogonal errors $A, B$ and $C$ must be significant enough to compensate for such overhead. Note also that our approach resembles triple modular redundancy but operates differently. Here, we exploit the error masking property in majority operators to enforce logic simplifications rather than covering potential hardware failures.

In the following, we present two methods for identifying advantageous triplets of orthogonal errors.

\section{B. Critical Voters Method}

A natural way to discover advantageous triplets of orthogonal errors is to analyze an MIG structure. We want to identify critical portions of an MIG to be simplified by these errors. To do so, we focus on nodes that have the highest impact on the final voting decision, i.e., influencing most a function computation. We call such nodes critical voters of an MIG. Critical voters can also be primary input themselves. To determine the critical voters, we rank MIG nodes based on a criticality metric. The criticality computation goes as follows. Consider a MIG node, say $m$. We label all MIG nodes whose computation depends on $m$. For all such nodes, we calculate the impact of $m$ by propagating a unit weight value from $m$ outputs

${ }^{1}$ For the sake of comprehension and conciseness, we present the theoretical concepts in an intuitive way. A formal treatment is directly derivable. 


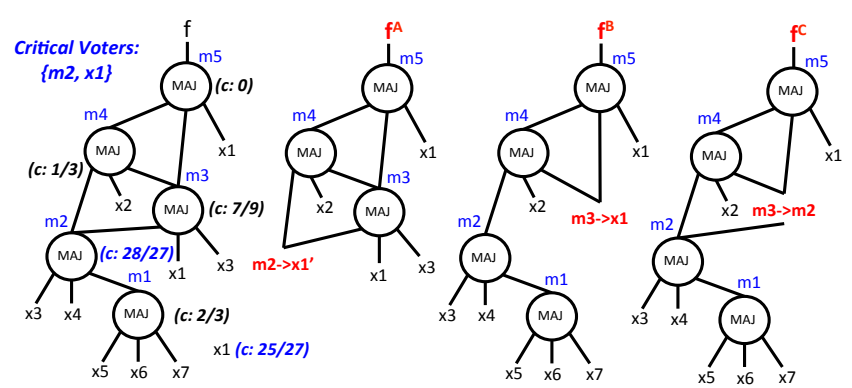

Fig. 2: Example of criticality computation and orthogonal errors.

up to the root with an attenuation factor of $1 / 3$ each time a majority node is encountered. We finally sum up all the values obtained and call this result criticality of $m$. Intuitively, MIG nodes with the highest criticality are critical voters. For the sake of clarity, we give an example of criticality computation in Fig. 2. Node $m 5$ has criticality of 0 , as it is the root. Node $m 4$ has criticality of $1 / 3$ (a unit weight propagated to $m 5$ and attenuated by $1 / 3$ ). Node $m 3$ has criticality of $1 / 3(m 4)+(1 / 3+1) / 3$ (direct and $m 4$ contribution to $m 5)$ which sums up to $7 / 9$. Node $m 2$ has criticality of $1 / 3(m 3)+4 / 9(m 4)+$ $7 / 27(m 5)$ which sums up to $28 / 27$. Node $m 1$ has criticality $1 / 3+$ criticality of $m 2$ attenuated by factor 3 which sums up to about $2 / 3$. Among the inputs, only $x 1$ has a notable criticality being $1 / 3(\mathrm{~m} 3)$ $+1 / 9(m 4)+(1 / 3+1 / 9+1) / 3(m 5)$ which sums up to $25 / 27$. Here the two elements with highest criticality are $m 2$ and $x 1$.

Given two critical voters $a$ and $b$ and the set of MIG nodes fed by both $a$ and $b$, say $\left\{c_{1}, c_{2}, \ldots, c_{n}\right\}$, an advantageous triplet of orthogonal errors is: $A$ : $a=b^{\prime}, B: c_{1}=a, c_{2}=a, \ldots, c_{n}=a$ and $C: c_{1}=b, c_{2}=b, \ldots, c_{n}=b$. Considering back the example in Fig. 2 the critical voters are $a=m 2$ and $b=x 1$ while $c_{1}=m 3$. Here, the pairwise orthogonal errors are $m 2=x 1^{\prime}(A), m 3=x 1$ $(B)$ and $m 3=m 2(C)$ as shown in Fig. 2. The actual orthogonality of $A, B$ and $C$ type of errors is proved in the following.

Theorem 3.2: Say $a$ and $b$ two critical voters in an MIG. Say $\left\{c_{1}, c_{2}, \ldots, c_{n}\right\}$ the set of MIG nodes fed by both $a$ and $b$ in the same polarity. The following errors are pairwise orthogonal: $A$ : $a=b^{\prime}$, $B: c_{1}=a, c_{2}=a, \ldots, c_{n}=a$ and $C: c_{1}=b, c_{2}=b, \ldots, c_{n}=b$.

Proof: Starting from an MIG $w$, we build the three faulty versions $w^{A}, w^{B}$ and $w^{C}$ as described above. We show that orthogonality holds for all 3 pairs. pair $\left(\boldsymbol{w}^{A}, \boldsymbol{w}^{B}\right)$ We need to show that $\left(w^{A} \oplus w\right) \cdot\left(w^{B} \oplus w\right)=0$. The element $w^{A} \oplus w$ implies $a=b$, being the difference between the original and the faulty one with $a=b^{\prime}$ $(a \neq b)$. The element $w^{B} \oplus w$ implies $c_{i} \neq a\left(c_{i}=a^{\prime}\right)$, being the difference between the original and the faulty one with $c_{i}=a$. However, if $a=b$ then $c_{i}$ cannot be $a^{\prime}$, because $c_{i}=M(a, b, x)=$ $M(a, a, x)=a \neq a^{\prime}$ by construction. Thus, the two elements cannot be true at the same time making $\left(w^{A} \oplus w\right) \cdot\left(w^{B} \oplus w\right)=0$. pair $\left(\boldsymbol{w}^{A}, \boldsymbol{w}^{C}\right)$ This case is symmetric to the previous one. pair $\left(\boldsymbol{w}^{B}, \boldsymbol{w}^{C}\right)$ We need to show that $\left(w^{B} \oplus w\right) \cdot\left(w^{C} \oplus w\right)=0$. As we deduced before, the element $w^{B} \oplus w$ implies $c_{i} \neq a\left(c_{i}=a^{\prime}\right)$. Similarly, the element $w^{C} \oplus w$ implies $c_{i} \neq b\left(c_{i}=b^{\prime}\right)$. By the transitive property of equality and congruence in the Boolean domain $c_{i} \neq a$ and $c_{i} \neq b$ implies $a=b$. However, if $a=b$, then $c_{i}=M(a, b, x)=M(a, a, x)=M(b, b, x)=a=b$ which contradicts both $c_{i} \neq a$ and $c_{i} \neq b$. Thus, the two elements cannot be true simultaneously making $\left(w^{B} \oplus w\right) \cdot\left(w^{C} \oplus w\right)=0$. Q.E.D.

Even though focusing on critical voters is typically a good strategy, sometimes other approaches can be also convenient. In the following, we present one of such substitute approaches.

\section{Input Partitioning Method}

As a complement to critical voters method, we propose a different way to derive advantageous triplets of orthogonal errors. In this case, we focus on the inputs rather than looking for internal MIG nodes. In particular, we search for inputs leading to advantageous simplifications when faulty. Similarly to the criticality metric in critical voters, we use here a decision metric, called dictatorship [18], to select the most profitable inputs. The dictatorship is the ratio of input patterns over the total $\left(2^{n}\right)$ for which the output assumes the same value of the selected input [18]. For example, in the function $f=(a+b) \cdot c$, the inputs $a$ and $b$ have equal dictatorship of 5/8 while input $c$ has an higher dictatorship of 7/8. The inputs with highest dictatorship are the ones where we want to insert logic errors. This is because they influence most a circuit functionality, and so also its structure. Considering back the example $f=(a+b) \cdot c$, suppose we are allowed to introduce a stuck at 0 error at one input. Appliying this error to $a$ or $b$ inputs (with low dictatorship) we reduce the complexity to a single gate $(a c$ or $b c)$. However, if we introduce the same error on the input $c$ (with high dictatorship) we further reduce the complexity just to a logic constant (0).

Exact computation of the dictatorship requires exhaustive simulation of an MIG structure, which is likely to be infeasible for practical functions of interest. Heuristic approaches to estimate dictatorship involve partial random simulation and graph techniques [18].

After dictatorship computation, we select a proper subset of the primary inputs. Next, for each selected input, we determine a condition that causes an error. We require these errors to be orthogonal. Since we operarte directly on the primary inputs, we divide the Boolean space into disjoint sub-sets that are natively orthogonal. As we need three errors, we need to consider at least three inputs to be made faulty, say $x, y$ and $z$. A possible division is the following: $\{x \neq y$, $\left.x=y=z, x=y=z^{\prime}\right\}$. The correspoding errors can be $A: x=y$ for $\{x \neq y\}, B: z=y^{\prime}$ when $x=y$ for $\{x=y=z\}$ and $C: z=y$ when $x=y$ for $\left\{x=y=z^{\prime}\right\}$. We formally prove that $A, B$ and $C$ are orthogonal errors hereafter.

Theorem 3.3: Consider the input division into $\{x \neq y, x=y=$ $\left.z, x=y=z^{\prime}\right\}$ in an MIG. Three errors $A, B$ and $C$ selectively affecting one subset but not the others are pairwise orthogonal.

Proof: To prove the theorem it is sufficient to show that the division $\left\{x \neq y, x=y=z, x=y=z^{\prime}\right\}$ is actually a partition of the whole Boolean space, i.e., a union of disjoint (non-overlapping) subsets. In Table I, all the eight possible $\{x, y, z\}$ combinations are

TABLE I: Input division into 3 pairwise disjoint sub-sets.

\begin{tabular}{|c|c|c|c|c|}
\hline sub-set & $x$ & $y$ & $z$ & $f$ \\
\hline$x=y=z$ & 0 & 0 & 0 & $f_{0}$ \\
\hline$x=y=z^{\prime}$ & 0 & 0 & 1 & $f_{1}$ \\
\hline$x \neq y$ & 0 & 1 & 0 & $f_{2}$ \\
\hline$x \neq y$ & 0 & 1 & 1 & $f_{3}$ \\
\hline$x \neq y$ & 1 & 0 & 0 & $f_{4}$ \\
\hline$x \neq y$ & 1 & 0 & 1 & $f_{5}$ \\
\hline$x=y=z^{\prime}$ & 1 & 1 & 0 & $f_{6}$ \\
\hline$x=y=z$ & 1 & 1 & 1 & $f_{7}$ \\
\hline
\end{tabular}

shown. The corresponding $\left\{x \neq y, x=y=z, x=y=z^{\prime}\right\}$ subsets are assigned in the left column. We visually see that all sub-sets are disjoint, i.e., they have no common input pattern. Moreover, all together, they form the whole Boolean space. Q.E.D.

So far, we shown how "safe error insertion" in MIGs can be accomplished by means of different techniques. In the rest of this paper, we will exploit the logic opportunities deriving from "safe error insertion" in MIG optimization.

\section{BoOleAN LOGIC OptimizATION IN MIG}

In this section, we propose Boolean optimization methods for MIGs by exploiting safe error insertion schemes. Our optimization procedures target depth and size reduction in MIGs. At the end of this section, we showcase our Boolean optimization capabilities for adder circuits. 


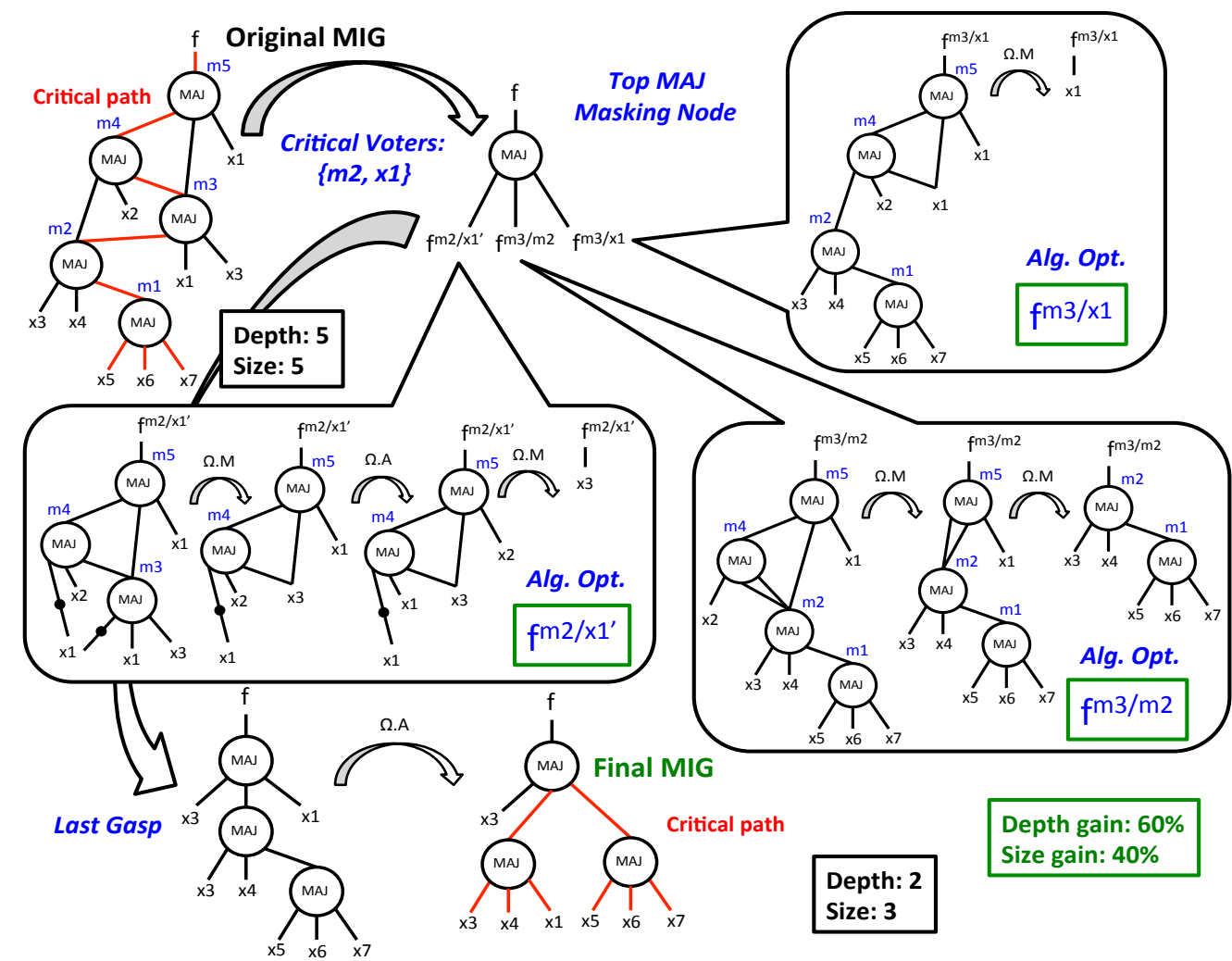

Fig. 3: MIG Boolean depth-optimization example based on critical voters errors insertion. Final depth reduction: $60 \%$, size reduction: $40 \%$.

\section{A. Depth-Oriented Boolean Methods}

The most intuitive way to exploit the voting resilience in MIGs is to reduce the number of levels. This is because the opening overhead of safe error insertion is just one additional level. Such extra level is usually well recovered during simplification and optimization of MIG faulty branches. For depth-optimization purposes, the critical voters method enables very good results. The reason is the following. Critical voters mostly appear on the critical path and reconverge on it. Thus, the possibility to insert simplifying errors on critical voters directly enables a strong reduction in the maximum number of levels.

Sometimes, using an actual MIG root as error insertion root requires an $3 \times$ size overhead whis is unpractical. In these cases, we bound the critical voters search to sub-MIGs partitioned on a depth criticality basis. Once the critical voters and a proper error insertion root have been identified, three faulty sub-MIG versions are generated as explained in the previous section. On these subMIGs, we want to reduce the logic height. We do so by running algebraic MIG optimization on them. Note that, in principle, also MIG Boolean methods can be re-used. This would correspond to a recursive Boolean optimization. However, it turned out during experimentation that algebraic optimizations already produce satisfactority results at the local level. Thus, it makes more sense to apply Boolean techniques iteratively on the whole MIG structure rather than recursively on the same logic portion.

At the end of the faulty branches optimization, the new MIG-roots must be given in input to a top majority voting node to re-establish the functional correctness. A last gasp of MIG algebraic optimization is convenient at this point, to take advantage of the simplification opportunities arosen from the faulty branches integration. The above described optimization strategy is summarized in Alg. 1.

For the sake of clarity, we comment on Boolean MIG-depth optimization with a simple example, reported in Fig. 3. First, the critical voters are searched and indetified, being in this example the input $x 1$ and the node $m 2$ (from Fig. 2). The proper error insertion

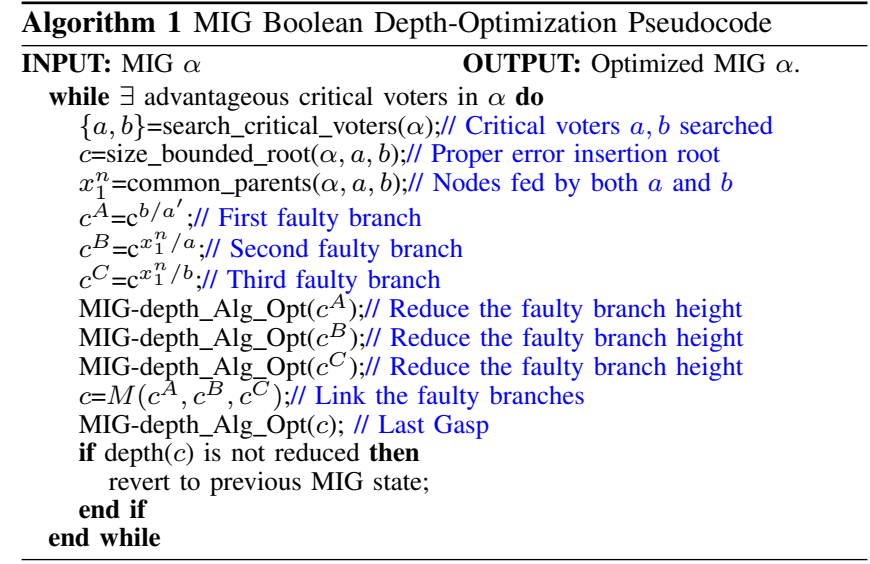

root in this small example is the MIG root itself. So, three different versions of the root $f$ are generated with errors $f^{m 2 / x 1^{\prime}}, f^{m 3 / m 2}$ and $f^{m 3 / x 1}$. Each faulty branch is handled by fast algebraic optimization to reduce its depth. The detailed algebraic optimization steps involved are shown in Fig. 3. The most common operation is $\Omega . M$ that directly simplifies the introduced errors. The optimized faulty branches are then linked together by a top fault-masking majority node. A last gasp of algebraic optimization on the final MIG structure further optimizes its depth. In summary, our MIG Boolean optimization techniques attains a depth reduction of $60 \%$ and, at the same time, a size reduction of $40 \%$. On the other hand, by running just algebraic optimization on this example a depth reduction of $20 \%$ is possible at a size overhead cost of $50 \%$.

\section{B. Size-Oriented Boolean Methods}

The voting resilience in an MIG can be also used to reduce its size. In this case, the branch triplication overhead imposes tight simplification requirements deriving from the inserted errors. In order 
to do so, we can still focus on critical voters and enforce more strict selection metrics. However, the benefit deriving from this approach is limited. A better solution is to change the type of error inserted and use the input partitioning method. Indeed, the input partitioning method focuses on the inputs that inflates most an MIG, and introduce selective simplification on them. The resulting Boolean optimization procedure is in principle identical to Alg. 1 but with depth techniques replaced by size techniques and critical voter search replaced by input partitioning methods. We do not discuss on the implementation details for MIG Boolean size optimization for the sake of brevity.

\section{Case Study: Adders Optimization}

Adders are hard to optimize circuits due to their inherent arithmetic nature. For this reason, they are good benchmarks to test the capabilities of logic optimization methods and associated tools. We bench our MIG Boolean depth optimization technique for different types of adders. We consider two, three and four operands adders, with bit widths ranging from 32 to 256 . Table II shows the optimization results. Our optimized MIG adders are 4 to $48 \times$ shorter than the original ones. In all cases, the optimized MIG structure resembles a carry-look ahead design which is known to be the most depth-efficient for adders. This is a remarkable results as standard synthesis engines cannot reach this level of automated optimization.

It is worth noticing that, even though very powerful, our Boolean MIG optimization is still an heuristic. This means that, on average, we get strong results but there is no guarantee on the degree of optimality. For example, the 2-operand 64 bit and 256 adders find early good critical voters enabling powerful depth minimization. On the other hand, 2-operand 32 bit and 128 adders do not find similar critical voters obtaining less depth reduction.

Original and our MIG-optimized Verilog files are downloadable at [19] for the sake of reproducibility.

TABLE II: Adder Optimization Results

\begin{tabular}{|c|c|c|c|c|c|c|}
\hline Adder type & Inputs & Outputs & \multicolumn{2}{c|}{ Original AIG } & \multicolumn{2}{c|}{ Optimized MIG } \\
\hline \multicolumn{3}{|l|}{} & Size & Depth & Size & Depth \\
\hline 2-op 32 bit & 64 & 33 & 352 & 96 & 610 & 12 \\
\hline 2-op 64 bit & 128 & 65 & 704 & 192 & 1159 & 11 \\
\hline 2-op 128 bit & 256 & 129 & 1408 & 384 & 14672 & 19 \\
\hline 2-op 256 bit & 512 & 257 & 2816 & 768 & 7650 & 16 \\
\hline 3-op 32 bit & 96 & 32 & 760 & 68 & 1938 & 16 \\
\hline 4-op 64 bit & 256 & 66 & 1336 & 136 & 2212 & 18 \\
\hline
\end{tabular}

V. EXPERIMENTAL RESULTS

In this section, we test the performance of our MIG Boolean optimization methods on academic and industrial benchmarks. We run pure logic optimization experiments and complete design experiments on a $22-\mathrm{nm}$ commercial ASIC flow.

\section{A. Methodology}

We developed a Majority-Logic manipulation Package (MLP) consisting of about $8 \mathrm{k}$ lines of $\mathrm{C}$ code. It embeds state-of art algebraic MIG optimization techniques [11] and the previously presented MIG Boolean optimization methods. As a global optimization flow, we focus on aggressive depth reduction interlaced with size recovery phases. For this purpose, we run algebraic optimization as long as improvements exist and then we run Boolean optimization to unlock further improvements. For our MIG Boolean depth-methods, we use critical voters search starting from tight selection constraints (enabling the largest advantage) and then decreasing till (i) a good pair of critical voters is found or (ii) a minimum threshold is reached. During size recovery, we employ Boolean methods based on input partitioning together with algebraic techniques. The MLP reads Verilog or AIGER format and writes back a Verilog description of the optimized MIG. We consider IWLS'05 Open Cores benchmarks and larger arithmetic HDL benchmarks (differential equation solvers, telecommunication units, sorters, specialized arithmetic units, etc.). All the input and output (Verilog) files from our experiments can be downloaded at [19], for the sake of reproducibility. In total, we optimized, and verified, $\sim 0.5$ million eq. gates over 27 benchmarks.

For the pure logic optimization experiments, we use as counterpart tool the ABC academic synthesizer [13], with delay oriented script if $-g$;iresyn. For the complete design flow experiments, we consider a state-of-art 22-nm commercial ASIC flow suite (logic synthesis + place $\&$ route). In this case, our MLP package operates as a front-end to the flow. As the circuit speed is our main design goal, we use an ultra-high delay-effort script in the commercial tools.

\section{B. Optimization Results}

Table III shows the results for MIG Boolean optimization. For the IWLS'05 and HDL arithmetic benchmarks, we see a total improvements in all size, depth and power activity metrics, w.r.t. to AIG optimized by ABC. Since depth was our main optimization target, we notice there the largest reduction. Considering the IWLS'05 benchmarks, that are large but not tall, in terms of number of levels, we see a $17.98 \%$ reduction. At the same time, the size and power are reduced by $12.65 \%$ and $10.00 \%$, respectively. Focusing on the arithmetic HDL benchmarks, we see a better depth reduction. Here, our MIG Boolean mehtodology enables a $26.69 \%$ depth reduction. At the same time, we reduce size and power by $7.7 \%$ and $0.1 \%$.

Table III shows that the runtime of our tool is competitive with that of ABC tool. This confirms the scalability of our methods, handling $100 \mathrm{k}$ equivalent gates in less than a minute, on a standard laptop.

Even though we do not use the same set of benchmarks in [11], we still want to provide a comparison between algebraic and Boolean MIG techniques. On average over our IWLS+HDL benchmarks, only about half of the reported improvements were possible just using algebraic techniques in our tool. However, this still does not directly relate to the numbers reported in [11]. For the sake of comparison, we optimize four relevant MCNC benchmarks also appearing in [11]: my_adder, alu4, clma and s38417. In [11], they have 19, 14, 42 and 22 number of levels, respectively. With our new MLP tool featuring Boolean optimization we lowered these numbers to 9, 11, 21 and 17, respectively. Also size and power metrics are lowered. These experiments can be downloaded at [19].

All MIG output Verilog files underwent formal verification experiments (ABC cec and Synopsys Formality) with success.

\section{ASIC Results}

Table IV shows the results for ASIC design (synthesis followed by place and route) at a commercial $22 \mathrm{~nm}$ technology node ${ }^{2}$. In total, we see that using our MIG optimizer as front-end to the ASIC design flow we enable better final circuits, in all area, delay and power metrics. For the delay, that was our critical design constraint, we observe an improvement of $15.07 \%$. This improvement is not as large as the one we saw at the logic optimization level. Indeed, some of that gain got absorbed by the interconnect overhead during physical design. However, we still see a coherent trend. Considering area and power we got reductions of $4.93 \%$ and $1.93 \%$, respectively.

In summary, using the MIG Boolean technology we observe consistent, and global, advantages over a state-of-art commercial design flow. It is worth noticing that we employed our method just as a front-end to an existing commercial flow. We foresee even better results by integrating MIG optimization inside the synthesis engine.

\section{Vi. Conclusions}

In this paper, we presented a Boolean logic optimization framework based on Majority-Inverter Graph (MIG). We proposed MIG optimization methods taking advatantage of the error masking property of majority operators. By inserting logic errors in an MIG, successively masked by majority nodes, we strongly simplified logic networks. Our Boolean methods are simple, yet powerful. Experiments combined with state-of-art MIG algebraic techniques shown tremendous results.

${ }^{2}$ Design tools and library names are omitted due to our license agreement. 
TABLE III: MIG Boolean Optimization Results

\begin{tabular}{|c|c|c|c|c|c|c|c|c|c|c|}
\hline \multirow{2}{*}{\multicolumn{2}{|c|}{ Open Cores IWLS'05 }} & \multicolumn{5}{|c|}{ MLP } & \multicolumn{4}{|c|}{$\mathrm{ABC}$} \\
\hline & & Size & Depth & Power & MAJ\% & Runtime & Size & Depth & Power & Runtime \\
\hline Benchmark & $\mathrm{I} / \mathrm{O}$ & & \multirow[b]{2}{*}{34} & \multirow[b]{2}{*}{$30 \mathrm{k}$} & \multirow[b]{2}{*}{15.02} & \multirow[b]{2}{*}{12.54} & \multirow[b]{2}{*}{44644} & \multirow[b]{2}{*}{47} & \multirow[b]{2}{*}{$32 \mathrm{k}$} & \multirow[b]{2}{*}{9.21} \\
\hline DSP & $4365 / 4145$ & & & & & & & & & \\
\hline ac97_ctrl & $2267 / 2262$ & 12006 & 8 & $10 \mathrm{k}$ & 22.14 & 9.76 & 14292 & 11 & $12 \mathrm{k}$ & 9.88 \\
\hline aes_core & $789 / 668$ & 20518 & 19 & $15 \mathrm{k}$ & 11.78 & 10.68 & 21543 & 22 & $14 \mathrm{k}$ & 8.21 \\
\hline des_area & $368 / 72$ & 4882 & 24 & $3 \mathrm{k}$ & 15.14 & 0.63 & 4858 & 28 & $2.9 \mathrm{k}$ & 1.08 \\
\hline des_perf & 9042/9038 & 81070 & 14 & $70 \mathrm{k}$ & 10.09 & 39.34 & 88317 & 17 & $69 \mathrm{k}$ & 22.92 \\
\hline ethernet & $10710 / 10728$ & 62301 & 17 & $35 \mathrm{k}$ & 20.77 & 20.28 & 86656 & 22 & $53 \mathrm{k}$ & 25.99 \\
\hline $\mathrm{i} 2 \mathrm{c}$ & $147 / 142$ & 1049 & 9 & $0.8 \mathrm{k}$ & 15.06 & 0.21 & 1136 & 10 & $0.8 \mathrm{k}$ & 0.06 \\
\hline mem_ctrl & $1204 / 1231$ & 9555 & 17 & $6 \mathrm{k}$ & 24.25 & 0.51 & 9396 & 28 & $5 \mathrm{k}$ & 0.26 \\
\hline pci_bridge32 & $3527 / 3534$ & 21170 & 17 & $15 \mathrm{k}$ & 36.92 & 3.88 & 23461 & 19 & $16 \mathrm{k}$ & 3.22 \\
\hline pci_spoci_ctrl & $89 / 80$ & 793 & 11 & $0.4 \mathrm{k}$ & 22.57 & 0.05 & 1291 & 13 & $0.7 \mathrm{k}$ & 0.02 \\
\hline sasc & $133 / 132$ & 661 & 6 & $0.6 \mathrm{k}$ & 15.58 & 0.22 & 753 & 8 & $0.7 \mathrm{k}$ & 0.07 \\
\hline simple_spi & $148 / 147$ & 976 & 8 & $0.8 \mathrm{k}$ & 18.65 & 0.15 & 1033 & 10 & $0.8 \mathrm{k}$ & 0.07 \\
\hline spi & $274 / 276$ & 4953 & 19 & $3 \mathrm{k}$ & 23.04 & 1.79 & 5548 & 21 & $3 \mathrm{k}$ & 1.85 \\
\hline ss_pcm & $106 / 98$ & 436 & 6 & $0.4 \mathrm{k}$ & 14.91 & 0.05 & 400 & 7 & $0.3 \mathrm{k}$ & 0.01 \\
\hline systemcaes & $930 / 819$ & 10599 & 27 & $8 \mathrm{k}$ & 31.11 & 11.21 & 12532 & 31 & $10 \mathrm{k}$ & 5.05 \\
\hline systemcdes & $314 / 258$ & 2936 & 19 & $2.4 \mathrm{k}$ & 13.11 & 3.62 & 3147 & 21 & $2.4 \mathrm{k}$ & 1.95 \\
\hline tv80 & $379 / 410$ & 8076 & 31 & $5 \mathrm{k}$ & 40.20 & 8.95 & 9494 & 36 & $6 \mathrm{k}$ & 3.22 \\
\hline usb_funct & $1894 / 1879$ & 14926 & 18 & $12 \mathrm{k}$ & 25.05 & 12.62 & 15644 & 20 & $13 \mathrm{k}$ & 9.34 \\
\hline usb_phy & $113 / 111$ & 439 & 6 & $0.4 \mathrm{k}$ & 10.25 & 0.04 & 478 & 7 & $0.4 \mathrm{k}$ & 0.11 \\
\hline \multicolumn{2}{|c|}{ IWLS'05 total } & 301027 & 310 & $217.80 \mathrm{k}$ & $372.39(19.59 \%)$ & 136.99 & 344623 & 378 & $242.00 \mathrm{k}$ & 102.45 \\
\hline \multicolumn{2}{|c|}{ Arithmetic HDL } & $\overline{\text { Size }}$ & $\overline{\text { Depth }}$ & 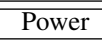 & MAJ\% & Runtime & $\overline{\text { Size }}$ & Depth & Power & Runtime \\
\hline MUL32 & $64 / 64$ & 9027 & 37 & $7.4 \mathrm{k}$ & 12.38 & 3.39 & 8630 & 43 & $7.2 \mathrm{k}$ & 1.80 \\
\hline sqrt32 & $32 / 16$ & 1923 & 170 & $1.7 \mathrm{k}$ & 26.00 & 1.20 & 1959 & 203 & $1.5 \mathrm{k}$ & 1.55 \\
\hline diffeq1 & $355 / 289$ & 33398 & 184 & $28 \mathrm{k}$ & 21.49 & 123.55 & 33632 & 303 & $26 \mathrm{k}$ & 18.91 \\
\hline $\operatorname{div} 16$ & $32 / 32$ & 2972 & 113 & $2.5 \mathrm{k}$ & 33.63 & 6.39 & 5016 & 137 & $3.6 \mathrm{k}$ & 2.21 \\
\hline hamming & $200 / 7$ & 2034 & 59 & $1.7 \mathrm{k}$ & 10.72 & 18.99 & 2717 & 75 & $1.6 \mathrm{k}$ & 2.31 \\
\hline MAC32 & $96 / 65$ & 10529 & 40 & $8.5 \mathrm{k}$ & 12.00 & 5.53 & 10320 & 70 & $8 \mathrm{k}$ & 7.65 \\
\hline metric_comp & $288 / 208$ & 18529 & 75 & $12 \mathrm{k}$ & 15.39 & 22.43 & 20821 & 112 & $13 \mathrm{k}$ & 10.22 \\
\hline revx & $20 / 25$ & 7625 & 146 & $5.5 \mathrm{k}$ & 15.63 & 12.33 & 10135 & 181 & $6.5 \mathrm{k}$ & 19.45 \\
\hline \multicolumn{2}{|c|}{ Arithmetic total } & 85997 & 824 & $\overline{767.30 \mathrm{k}}$ & $146.70(18.33 \%)$ & 193.81 & 993230 & 1124 & $\overline{667.40 \mathrm{k}}$ & 264.10 \\
\hline
\end{tabular}

TABLE IV: MIG 22-nm ASIC Design Results

\begin{tabular}{|c|c|c|}
\hline Benchmark & MLP+ASIC flow & ASIC flow \\
\hline & $\mu m^{2} / n s / \mu W$ & $\mu m^{2} / n s / \mu W$ \\
\hline MUL32 & $1841.76 / 0.52 / 1.82$ & $1958.81 / 0.57 / 1.79$ \\
\hline diffeq1 & $3992.49 / \mathbf{2 . 8 5} / 4.57$ & $3908.15 / 3.38 / 4.50$ \\
\hline hamming & $361.50 / 0.87 / 0.56$ & $395.00 / 0.98 / 0.59$ \\
\hline $\operatorname{div} 16$ & $720.45 / 1.56 / 0.27$ & $950.07 / 1.83 / 0.35$ \\
\hline sqrt32 & 505.78/1.97/0.50 & $455.95 / 2.20 / 0.48$ \\
\hline DSP & $7123.410 .47 / 2.45$ & $7119.60 / 0.49 / 2.51$ \\
\hline ac97_ctrl & $2295.09 / \mathbf{0 . 1 0} / 0.53$ & $2398.90 / 0.12 / 0.55$ \\
\hline aes_core & $4597.55 / \mathbf{0 . 2 3} / 1.54$ & $5272.32 / 0.25 / 1.55$ \\
\hline des_area & $956.04 / 0.32 / 0.54$ & $1084.60 / 0.36 / 0.53$ \\
\hline des_perf & $14790.03 / \mathbf{0 . 1 8} / 9.75$ & $15211.80 / 0.20 / 9.76$ \\
\hline ethernet & $11235.40 / \mathbf{0 . 1 8} / 1.31$ & $10950.19 / 0.23 / 1.39$ \\
\hline $\mathrm{i} 2 \mathrm{c}$ & $210.13 / \mathbf{0 . 1 0} / 0.04$ & $210.04 / 0.11 / 0.04$ \\
\hline mem_ctrl & $1418.22 / 0.26 / 0.24$ & $1418.22 / 0.34 / 0.25$ \\
\hline pci_bridge 32 & $3209.76 / \mathbf{0 . 2 5} / 0.68$ & $3250.08 / 0.27 / 0.70$ \\
\hline pci_spoci_ctrl & 159.34/0.16/0.08 & 177.47/0.16/0.09 \\
\hline sasc & $125.12 / 0.08 / 0.02$ & $139.98 / 0.10 / 0.02$ \\
\hline simple_spi & $176.34 / \mathbf{0 . 1 2} / 0.04$ & $163.72 / 0.15 / 0.04$ \\
\hline spi & $623.16 / 0.24 / 0.21$ & $550.95 / 0.30 / 0.18$ \\
\hline ss_pcm & $85.33 / \mathbf{0 . 0 8} / 0.02$ & $89.23 / 0.08 / 0.02$ \\
\hline systemcaes & $1380.07 / 0.31 / 0.54$ & $1322.87 / 0.37 / 0.51$ \\
\hline systemcdes & $665.01 / \mathbf{0 . 2 6} / 0.39$ & $731.71 / 0.30 / 0.43$ \\
\hline tv80 & $1342.52 / 0.39 / 0.34$ & $1295.10 / 0.49 / 0.37$ \\
\hline usb_funct & $2388.53 / 0.25 / 0.69$ & $2359.15 / 0.26 / 0.68$ \\
\hline usb_phy & $111.15 / 0.05 / 0.02$ & $115.73 / 0.07 / 0.02$ \\
\hline MAC32 & $2287.50 / \mathbf{0 . 4 8} / 1.74$ & $2502.68 / 0.61 / 1.92$ \\
\hline metric_comp & $3975.97 / 1.18 / 1.21$ & $4606.42 / 1.41 / 1.41$ \\
\hline revx & 1506.39/1.92/1.76 & 1931.07/2.48/1.81 \\
\hline Total & 67085.01/15.38/31.86 & $70569.81 / \mathbf{1 8 . 1 1 / 3 2 . 4 9}$ \\
\hline
\end{tabular}

For example, when targeting depth reduction, our MIG optimizer transformed ripple carry adders into a carry look-ahead ones. Over IWLS'05 and arithmetic HDL benchmarks, we reduced the logic network depth by $17.98 \%$ and $26.69 \%$, respectively, while also improving size and power metrics. Employed as a front-end to a delay-critical 22-nm ASIC flow (logic synthesis + physical design) our MIG optimizer reduced the average delay/area/power by $(15.07 \%$, $4.93 \%, 1.93 \%$ ), over 27 academic and industrial benchmarks, as compared to a leading commercial ASIC flow.

\section{ACKNOWLEDGEMENTS}

This research was supported by ERC-2009-AdG-246810.

\section{REFERENCES}

[1] A. Mishchenko, et al., Using simulation and satisfiability to compute flexibilities in Boolean networks IEEE TCAD 25.5 (2006): 743-755.

[2] A. Mishchenko, et al., Scalable don't-care-based logic optimization and resynthesis, ACM TRETS 4.4 (2011): 34

[3] C. Yang, et al., BDS: A BDD-based logic optimization system, IEEE TCAD 21.7 (2002): 866-876.

[4] N. Vemuri, et al., BDD-based logic synthesis for LUT-based FPGAs, ACM TODAES 7.4 (2002): 501-525.

[5] L. Amarú, et al., BDS-MAJ: a BDD-based logic synthesis tool exploiting majority logic decomposition, Proc. DAC'13.

[6] R.K. Brayton, et al., The Decomposition and Factorization of Boolean Expressions, Proc. ISCAS'82.

[7] R.K. Brayton, et al., Factoring logic functions, IBM Journal of Research and Development 31.2 (1987): 187-198.

[8] R.K. Brayton, et al., MIS: A multiple-level logic optimization system, IEEE TCAD 6.6 (1987): 1062-1081.

[9] E. Sentovich, et al., SIS: A System for Sequential Circuit Synthesis, ERL, Dept. EECS, Univ. California, Berkeley, UCB/ERL M92/41, 1992.

[10] R.K. Brayton, Multilevel logic synthesis, Proc. IEEE78.2(1990):264-300.

[11] L. Amarú, et al., Majority-Inverter Graph: A Novel Data-Structure and Algorithms for Efficient Logic Optimization, Proc. DAC'14.

[12] G. De Micheli, Synthesis and Optimization of Digital Circuits, McGrawHill, New York, 1994.

[13] ABC synthesis tool - http://www.eecs.berkeley.edu/ alanmi/abc/.

[14] John R. Isbell, Median algebra, Trans. Amer. Math. Soc., 319-362, 1980.

[15] D. Knuth, The Art of Computer Programming, Volume 4A, Part 1, New Jersey: Addison-Wesley, 2011

[16] G. Birkhoff, Lattice Theory, Amer. Math. Soc., New York, 1967

[17] S. Muroga, et al., The transduction method-design of logic networks based on permissible functions, IEEE TCOMP, 38.10 (1989): 1404-1424.

[18] M. Parnas, et al., Proclaiming dictators and juntas or testing boolean formulae, Combinatorial Optimization, Springer, 2001. 273-285.

[19] Majority-Inverter Graphs circuits available at http://lsi.epfl.ch/MIG 